\title{
Die Notwendigkeit unabhängiger klinischer Studien aus der Sicht des Instituts für Qualität und Wirtschaftlichkeit im Gesundheitswesen
}

\author{
Stefan Lange Jürgen Windeler \\ Institut für Qualität und Wirtschaftlichkeit im Gesundheitswesen, Köln, Deutschland
}

Schlüsselwörter

Klinische Studien - Klinische Forschung ·

Patientenrelevanz · Interessenkonflikte · Unabhängigkeit

\section{Zusammenfassung}

Die Ergebnisse klinischer, möglichst randomisierter kontrollierter Studien (RCT) bilden das Rückgrat von Entscheidungen zur Zulassung von Arzneimitteln und von Nutzenbewertungen medizinischer Methoden. Während Zulassungsstudien von Arzneimitteln häufig zumindest einige der für eine Nutzenbewertung relevanten Fragen beantworten, ist die Situation bei nichtmedikamentösen therapeutischen Methoden und diagnostischen Verfahren eine gänzlich andere, weil es hier keine vergleichbar hohen Anforderungen an eine Markteinführung gibt. Insgesamt muss festgestellt werden, dass es in der Vergangenheit für Hersteller oder Anbieter von medizinischen Methoden keine ausreichenden (finanziellen) Anreize gab und auch weiterhin nicht gibt, klinische Studien zum patientenrelevanten Nutzen durchzuführen, sowohl bei Arzneimitteln als auch insbesondere im nichtmedikamentösen Bereich. Daraus resultiert eine Lücke von Studien, die mit ausreichender Ergebnissicherheit in einem angemessenen Vergleich Daten zum patientenrelevanten Nutzen bzw. Zusatznutzen einer medizinischen Methode liefern. In diesem Zusammenhang ist es zweitrangig, ob es sich dabei um "unabhängige" Studien handelt, zumal "Abhängigkeiten" nie ausgeschlossen werden können und dort, wo sie nicht durch die Deklaration eines Sponsorings leicht erkennbar sind, besonders problematisch werden können. Das Institut für Qualität und Wirtschaftlichkeit im Gesundheitswesen (IOWiG) ist gerne bereit und dabei, zusammen mit Interessierten dafür zu werben, dass für wichtige Studienprojekte von klinischen Forschergruppen, die die Kriterien einer qualitativ hochwertigen patientenorientierten klinischen Studie zu einer relevanten Fragestellung erfüllen, angemessene Fördermöglichkeiten geschaffen werden.

\section{Keywords}

Clinical trials - Clinical research - Relevance for patients . Conflicts of interest $\cdot$ Independence

\section{Summary}

The Necessity of Independent Clinical Trials from the Perspective of the Institute of Quality and Efficiency in Health Care

The results of clinical, preferably randomized controlled trials (RCTs) form the backbone of drug approval decisions and benefit assessments of medical interventions. Whereas drug approval studies often answer at least some of the relevant questions posed in a benefit assessment, the situation is totally different for non-drug treatments and diagnostic tests, as the requirements for market entry are not as high in these fields. Overall it must be concluded that in the past and up to the present time there have been insufficient (financial) incentives for manufacturers or providers of medical interventions to conduct clinical trials concerning patient-relevant benefits both in the field of drugs and particularly in non-drug interventions. This has led to a lack of studies that, in an appropriate comparison with sufficient certainty of results, provide data on the patient-relevant benefits or added benefits of a medical intervention. In this context, it is secondary whether these are 'independent' studies, the more so as 'dependencies' can never be excluded and can become especially problematic in cases where they are not easily recognized by declaration of sponsorship. The Institute of Quality and Efficiency in Health Care (IOWiG) is willing to promote the creation of appropriate funding opportunities for important study projects of clinical research groups fulfilling the criteria for a high-quality patient-oriented clinical trial on a relevant research question, and is currently doing so together with interested parties.

\section{KARGER \\ Fax +497614520714 \\ Information@Karger.com}

www.karger.com (c) 2013 S. Karger GmbH, Freiburg

$0378-584 \mathrm{X} / 13 / 3614-0009 \$ 38.00 / 0$

Accessible online at:

www.karger.com/onk
PD Dr. med. Stefan Lange

Institut für Qualität und Wirtschaftlichkeit im Gesundheitswesen

Im Mediapark 8 (KölnTurm), 50670 Köln, Deutschland

stefan.lange@iqwig.de 


\section{Einleitung}

Seit Jahrzehnten ist anerkannt, dass die Ergebnisse klinischer Studien, möglichst randomisierter kontrollierter Studien (RCT), Voraussetzung für Zulassungsentscheidungen von Arzneimitteln sind [1]. Innerhalb der letzten 10 Jahre sind die Ergebnisse von RCT sowohl international als auch national zudem wegweisend und erforderlich geworden für Fragen und Entscheidungen zur Erstattungsfähigkeit und angemessenen Preisgestaltung von Arzneimitteln auf der Basis von Nutzen- und Kosten-Nutzen-Bewertungen. In Deutschland ist dies im Fünften Buch des Sozialgesetzbuches (SGB V) verankert, was die praktische Bedeutung dieses Standards unterstreicht [2, 3]. Die Regelungen und Anforderungen führen dazu, dass solche Studien in großer Zahl durchgeführt werden, und es stellt sich damit die Frage, welche Lücke besteht, die durch «unabhängige klinische Studien» gefüllt werden soll.

\section{Status quo Arzneimittel}

Die Forderung nach unabhängigen klinischen Studien impliziert, dass gegenwärtig zumindest ein großer Teil dieser Studien «abhängig» durchgeführt wird. Damit ist zumeist gemeint, dass Studien zu Arzneimitteln im Wesentlichen durch die pharmazeutische Industrie finanziert sind und damit deren Interessen bedient werden sollen. Solche Interessen können augenfällig und akzeptabel sein, nämlich dass nach jahrelangen Forschungsanstrengungen ein «gutes» Arzneimittel auch die Zulassung für den Markteintritt und im Markt dann einen fairen Preis erhält. Sie können aber auch inakzeptabel und ethisch fragwürdig sein, wenn ein Arzneimittel besser dargestellt werden soll, als es eigentlich ist. Die Mechanismen, wie Letzteres in Studien erreicht werden kann, sind bekannt und zahlreich [4]. Sie sind der häufigste Grund für den Ruf nach unabhängigen klinischen Studien, von denen man erwartet, dass sie faire Vergleiche und unverzerrte Aussagen sicherstellen.

Darüber hinaus beantworten viele Studien, speziell die mit dem Ziel einer Zulassung durchgeführten, nur die für diesen Zweck relevanten Fragen nach Sicherheit und Wirksamkeit eines Arzneimittels. Weitergehende Aspekte, die für die Bewertung eines patientenrelevanten Nutzens und insbesondere des Zusatznutzens von Arzneimitteln wesentlich sind, werden durch Zulassungsstudien häufig nicht oder allenfalls indirekt erfasst. So sind etwa Wirksamkeitskriterien (z.B. Tumorprogression anhand von bildgebenden Verfahren oder Laborwerten) nicht gleichbedeutend mit Nutzenkriterien (Mortalität, Morbidität und Lebensqualität). Und der Nachweis der Wirksamkeit sagt in aller Regel noch nichts darüber aus, wie ein Arzneimittel gegen den besten verfügbaren Therapiestandard abschneidet, was also sein Zusatznutzen ist. In Deutschland befinden sich z.B. gegenwärtig mindestens 6 Präparate zur
Behandlung des fortgeschrittenen, metastasierten Nierenzellkarzinoms auf dem Markt, ohne dass dazu ausreichend direkt vergleichende Studien vorliegen.

Die Situation für Arzneimittel ist dabei noch vergleichsweise gut. Immerhin gibt es hohe Anforderungen an die $\mathrm{Zu}$ lassung, und Zulassungsstudien können häufig zumindest einige der für die Nutzenbewertung relevanten Fragen beantworten. Ganz anders sieht es demgegenüber für nichtmedikamentöse therapeutische Methoden aus, von diagnostischen Verfahren ganz zu schweigen.

\section{Status quo nichtmedikamentöser Verfahren}

Nichtmedikamentöse therapeutische Methoden lassen sich in solche unterscheiden, bei denen maßgeblich ein Medizinprodukt beteiligt ist, und solche, bei denen dies nicht der Fall ist. Für Medizinprodukte gibt es zwar ein Regelwerk, das den Marktzugang regelt, die Anforderungen sind aber bei weitem geringer als diejenigen, die an die Zulassung von Arzneimitteln geknüpft sind [5]. So muss neben der Sicherheit der Medizinprodukte lediglich ihre Funktionstauglichkeit nachgewiesen werden, also dass z.B. ein Bestrahlungsgerät tatsächlich Strahlen emittiert und dass diese Strahlen grundsätzlich dazu in der Lage sind, beispielsweise Tumorzellen zu zerstören, oder dass ein Bildgebungsverfahren dazu in der Lage ist, mit ausreichender Genauigkeit Gewebestrukturen zu erkennen. Die Sicherheit in der Anwendung wird aber ebenso wenig geprüft wie die zentrale Frage, ob sich die Funktionstauglichkeit in einem Effekt im Sinne der Wirksamkeit oder gar in einem patientenrelevanten Nutzen niederschlägt. Entsprechende Nachweise müssen für die Markteinführung nicht erbracht werden. Wozu diese Praxis führen kann, hat das Beispiel der intrakraniellen Stents gezeigt: Eine Fallserie mit 45 Patienten und optimistisch stimmenden Ergebnissen führte zur Markteinführung [6], eine danach durchgeführte randomisierte Studie im Vergleich zu einer aggressiven medikamentösen Therapie wurde abgebrochen, nachdem eine Erhöhung schwerwiegender Ereignisse (Schlaganfall oder Tod) um 250\% in der Stent-Gruppe innerhalb der ersten 30 Tage beobachtet werden musste [7]. Der Begriff der «klinischen Prüfung» aus dem Medizinprodukterecht hat allenfalls marginal etwas mit dem gleichnamigen Begriff aus dem Arzneimittelrecht zu tun und erfordert keine aussagefähigen Studien, insbesondere keine RCT.

Nichtmedikamentöse therapeutische oder präventive Methoden, bei denen Medizinprodukte keine maßgebliche Rolle spielen, entziehen sich immer noch jeglicher Regulation im Sinne von Anforderungen für den Marktzugang. Beispielhaft seien Methoden aus dem Heilmittelbereich oder der Psychotherapie, aber auch zahlreiche chirurgische Verfahren oder präventive Maßnahmen insbesondere im Hinblick auf eine sogenannte gesunde Lebensführung genannt. Klinische Studien in diesen Bereichen sind zum Zeitpunkt ihrer Propagie- 
Tab. 1. Durch Beschlüsse des G-BA initiierte RCT

\begin{tabular}{|c|c|c|c|}
\hline Studie(n) & Fragestellung & Förderer & Status \\
\hline Gerdesmeyer et al., 2003 [10] & $\begin{array}{l}\text { extrakorporale Stoßwellenlithotripsie bei } \\
\text { chronisch kalzifizierender Tendinitis der } \\
\text { Rotatorenmanschette }\end{array}$ & $\begin{array}{l}\text { Deutsche Gesellschaft für } \\
\text { Orthopädie und orthopädische } \\
\text { Chirurgie, Hersteller }\end{array}$ & abgeschlossen \\
\hline BP-BVDD [11] & $\begin{array}{l}\text { asynchrone Balneophototherapie bei } \\
\text { Psoriasis }\end{array}$ & $\begin{array}{l}\text { Bundesverband Deutscher } \\
\text { Dermatologen }\end{array}$ & abgeschlossen \\
\hline TOMESA [12] & $\begin{array}{l}\text { synchrone Balneophototherapie bei } \\
\text { Psoriasis und atopischer Dermatitis }\end{array}$ & AOK Bayern & abgeschlossen \\
\hline GERAC $[13,14]$ & Akupunktur bei chronischen Schmerzen & Primärkassen der GKV & abgeschlossen \\
\hline ART $[15,16]$ & Akupunktur bei chronischen Schmerzen & Ersatzkassen der GKV & abgeschlossen \\
\hline DiaFu [17] & $\begin{array}{l}\text { Vakuumversiegelungstherapie von } \\
\text { Wunden - diabetische Fußulzera }\end{array}$ & GKV, Hersteller & Rekrutierung begonnen \\
\hline VAC2010-56 [18] & $\begin{array}{l}\text { Vakuumversiegelungstherapie von Wunden } \\
\text { - subkutane abdominelle Wundheilungs- } \\
\text { störungen nach chirurgischem Eingriff }\end{array}$ & GKV, Hersteller & Rekrutierung begonnen \\
\hline ETAL-1 [19] & $\begin{array}{l}\text { Stammzelltransplantation bei akuter } \\
\text { myeloischer Leukämie in erster Remission } \\
\text { mit Standardrisiko }\end{array}$ & BMBF & Rekrutierung begonnen \\
\hline PREFERE [20] & $\begin{array}{l}\text { Therapie des lokal begrenzten } \\
\text { Prostatakarzinoms }\end{array}$ & Krebshilfe, GKV & Rekrutierung beginnt 2013 \\
\hline
\end{tabular}

AOK = Allgemeine Ortskrankenkasse; GKV = gesetzliche Krankenversicherungen; BMBF = Bundesministerium für Bildung und Forschung.

rung sehr selten und in der Regel allein das Ergebnis akademischen Interesses.

Für diagnostische Methoden hat sich zwar bei anwendungsorientierten Methodikern und in den gesamten weltweiten Health Technology Assessment(HTA)- und Leitlinienaktivitäten die ebenfalls jahrzehntealte Erkenntnis durchgesetzt, dass Genauigkeit (Accuracy) und Informationsgewinn allein keinen patientenrelevanten Nutzen ausmachen [8]. In der klinischen Forschung ist diese Erkenntnis jedoch immer noch nicht ausreichend angelangt und Studien, die tatsächlich den patientenrelevanten Nutzen von Informationsgewinn und daraus resultierender (präventiver oder therapeutischer) Konsequenz prüfen, sind noch nicht die Regel. Da es sich bei diagnostischen Methoden zumeist um Medizinprodukte (oder um In-vitro-Diagnostika) handelt, existieren auch hier keine den patientenrelevanten Nutzen betreffenden Hürden für einen Marktzugang.

Außerhalb von Arzneimitteln existieren somit weder regulatorische Anforderungen noch andere finanzielle Anreize für die Durchführung klinischer Studien im Hinblick auf den Marktzugang. Und auch für den Zugang ins System der Gesetzlichen Krankenversicherungen (GKV) bestehen solche Anreize nur bedingt, da im stationären Sektor der sogenannte Verbotsvorbehalt gilt, der besagt, dass Leistungen, für die ein Marktzugang besteht, erbracht werden dürfen, so lange sie nicht durch den Gemeinsamen Bundesausschuss (G-BA) ausgeschlossen («verboten») werden. Der Verbotsvorbehalt ist durch das GKV-Versorgungsstrukturgesetz noch einmal akzentuiert worden, indem in Analogie zur Regelung bei Arzneimitteln (in § 92 SGB V) als Voraussetzung für einen Aus- schluss nicht allein der fehlende Nutzennachweis ausreicht. Es darf vielmehr auch kein «Potenzial» bestehen: «Ergibt die Überprüfung (durch den G-BA), dass der Nutzen einer Methode nicht hinreichend belegt ist und sie nicht das Potenzial einer erforderlichen Behandlungsalternative bietet, insbesondere weil sie schädlich oder unwirksam ist, erlässt der Gemeinsame Bundesausschuss eine entsprechende Richtlinie, wonach die Methode im Rahmen einer Krankenhausbehandlung nicht mehr zulasten der Krankenkassen erbracht werden darf» [9].

Soweit die Einführung des Verbotsvorbehalts im Jahr 2000 auch darauf abzielte, durch ungehinderte Anwendung von Methoden im Krankenhaus Erkenntnisse über ihren Nutzen zu gewinnen, kann festgestellt werden, dass dieses Ziel vollständig verfehlt wurde. Insofern ist es folgerichtig, dass durch die Einführung einer neuen Erprobungsregelung (§ 137e SGB V) nun Möglichkeiten und Anreize für das Durchführen von Studien geschaffen wurden. So kann der G-BA Richtlinien zur Erprobung einer neuen Methode beschließen, wenn für solche Methoden zwar der Nutzen (noch) nicht nachgewiesen ist, immerhin aber das Potenzial für einen Nutzen festgestellt werden kann. Was diese Regelungen in der Praxis bewirken, bleibt abzuwarten.

Für den ambulanten (vertragsärztlichen) Sektor ist die Situation genau umgekehrt: Der Nutzen einer Methode muss nachgewiesen sein, damit die Erstattungsfähigkeit positiv bewertet werden kann (sogenannter Erlaubnisvorbehalt). Im Gegensatz zum Verbotsvorbehalt hat diese Regelung in der Vergangenheit die Durchführung von aussagefähigen Studien stimuliert. Nach negativen oder Aussetzungsbeschlüssen des 
G-BA (unter anderem nach entsprechenden Empfehlungen des Instituts für Qualität und Wirtschaftlichkeit im Gesundheitswesen (IQWiG)) bzw. seiner Vorläuferinstitutionen wurden Studien initiiert und durchgeführt, die den Nutzen von Methoden untersuchen oder untersucht haben (Tab. 1). Diese Studien belegen im Übrigen eine bemerkenswerte Förder(er)vielfalt auch in Deutschland.

Neben Arzneimitteln und Medizinprodukten im eigentlichen Sinne gibt es noch «Arzneimittel für neuartige Therapien» (engl.: advanced therapy medicinal products (ATMP)). Darunter sind unter anderem Gentherapien, somatische Zelltherapien und biotechnologisch hergestellte Gewebeprodukte (engl.: tissue-engineered products (TEP)) zu verstehen, für die erst im Jahre 2007 eine europäische Regulation für die Zulassung verabschiedet wurde [21]. Davor konnten entsprechende Produkte ohne Zulassungsverfahren auf den Markt, sprich an den Patienten, gebracht werden. Gegenwärtig besteht für ATMP noch eine Übergangsphase, die Ende 2012 ausgelaufen ist. Bis August 2012 wurden lediglich 2 Produkte (ein TEP sowie ein gentherapeutisches Arzneimittel) nach den neuen Regularien zugelassen [22].

\section{Fehlende Anreize}

Es kann also festgestellt werden, dass es in der Vergangenheit für Hersteller oder Anbieter von medizinischen Methoden keine ausreichenden (finanziellen) Anreize gab und zweifelsohne noch gibt, klinische Studien zum patientenrelevanten Nutzen durchzuführen, sowohl bei Arzneimitteln als auch insbesondere im nichtmedikamentösen Bereich. Diese Lücken wurden und werden zwar teilweise durch akademisch initiierte Studien gefüllt, man denke hier z.B. an die zahlreichen nationalen und internationalen Studiengruppen aus der (Hämato-)Onkologie. Dennoch bleiben viele Fragen unbeantwortet, weil auch andere Anreize nicht bestehen. Neben dem Umstand, dass öffentliche Fördermittel für eine patientenorientierte klinische Forschung bei weitem nicht ausreichen, hatte und hat diese Forschungsorientierung keinen großen Stellenwert im Sinne von Anerkennung und Karrierechancen an deutschen Universitäten [23]. Auch das Verständnis von patientenorientierter klinischer Forschung als Frage nach einer direkten Beeinflussung der zentralen patientenrelevanten Domänen Mortalität, Morbidität und Lebensqualität ist im akademischen Bereich in der Vergangenheit nicht weit verbreitet gewesen. So hält sich hartnäckig die Vorstellung, pathophysiologische Surrogate seien ausreichend für den Nutzennachweis. Aktuelle Entscheidungen im Hinblick auf die Förderung von medizinischen Großprojekten scheinen die einseitige Allokation von Mitteln zu bestätigen, insbesondere wenn man sie in Relation zu den - inzwischen immerhin vorhandenen - Fördermöglichkeiten für patientenorientierte Forschung setzt. Hier haben erst zahlreiche Reformen im deutschen Gesundheitswesen zu einem gewissen Umdenken geführt. Stichworte wie evidenzbasierte Medizin sowie die Orientierung an Mortalität, Morbidität und Lebensqualität sind inzwischen fest im SGB V verankert. Dass Mitarbeiter des IQWiG im Rahmen dieses Themenheftes dazu aufgefordert wurden, einen Beitrag zur Notwendigkeit unabhängiger klinischer Studien zu verfassen, mag als weiteres Indiz für dieses Umdenken gedeutet werden.

\section{Beseitigen «unabhängige» Studien das Problem?}

Wir, die Patienten, die klinischen Anwender, das «System», brauchen Erkenntnisse aus Studien. Dabei ist es das primäre Interesse, dass es sich um «gute» klinische Studien handelt, die Fragen zum patientenrelevanten Nutzen adressieren. Studien müssen nicht zwangsläufig «unabhängig» sein, um «gut» zu sein, zumal sich die Frage stellt, was eigentlich «Unabhängigkeit» in diesem Zusammenhang genau bedeuten soll. Fokussiert man auf finanzielle Interessen im Zusammenhang mit dem Nutzennachweis für medizinische Methoden, bestehen diese nicht nur bei Herstellern von Arzneimitteln und Medizinprodukten, sondern selbstverständlich auch bei deren Anwendern, jedenfalls dann, wenn Vergütungssysteme existieren, die in erster Linie die Anwendung von medizinischen Methoden honorieren. Des Weiteren bestehen finanzielle Interessen, wenn Gehälter und Ausstattung an Universitäten von erfolgreicher Drittmitteleinwerbung abhängig gemacht werden und diese Drittmitteleinwerbung ihrerseits «erfolgreiche» Forschung und Publikationstätigkeit voraussetzt, die in der Vergangenheit häufig mit positiven Ergebnissen gleichgesetzt wurden. Und natürlich besteht eine «Abhängigkeit» auch im Sinne eines immateriellen Interessenkonflikts, wenn Forscherinnen und Forscher sich jahrelang mit einem bestimmten medizinischen Gebiet und spezifischen Hypothesen beschäftigen oder sich in der Anwendung von Methoden engagieren und diese Hypothesen oder Methoden dann auf den Prüfstand gestellt werden sollen.

Gerade weil «Abhängigkeiten» nie ausgeschlossen werden können und dort, wo sie nicht durch die Deklaration eines Sponsorings leicht erkennbar sind, besonders problematisch werden können, ist nicht die (finanzielle) «Unabhängigkeit», sondern ganz zentral die Qualität einer Studie von Interesse. Aber was macht nun «gute» klinische Studien aus? Diese Frage ist relativ leicht zu beantworten: Es muss sich um Studien handeln, die mit ausreichender Ergebnissicherheit in einem angemessenen Vergleich Daten zum patientenrelevanten Nutzen bzw. Zusatznutzen einer medizinischen Methode liefern.

Ergebnissicherheit wird im Wesentlichen durch drei Aspekte determiniert: das Verzerrungspotenzial (qualitative Ergebnissicherheit), die Präzision (Fallzahl, quantitative Ergebnissicherheit) und die Größe eines (beobachteten) Unterschieds. Während sich die beiden ersten Aspekte durch das Studiendesign beeinflussen lassen, reflektiert der dritte As- 
pekt eine Eigenschaft der medizinischen Methode, die es zu prüfen gilt. Die Präzision und die erwartete Größe eines Unterschieds stehen dabei in einem gewissen Spannungsverhältnis, wenn auf Basis statistischer Verfahren der Fallzahlplanung nach einer möglichst «effizienten» Fallzahl gesucht wird. Je größer nämlich ein Unterschied tatsächlich ist, umso geringer ist die Fallzahl, die benötigt wird, um überhaupt irgendeinen Unterschied (jenseits der Null-Differenz) nachzuweisen, was immer noch dem üblichen statistischen Vorgehen entspricht. Dies impliziert aber eine gleichmäßig niedrige Präzision (jedenfalls, wenn die Erwartung in etwa der Realität entspricht). Das Effizienzargument wird auch aus einer ethischen Perspektive ins Feld geführt, dass nämlich aus ethischer Sicht Patienten nicht mehr einer experimentellen Situation ausgesetzt werden dürften, wenn sich eine der Alternativen als überlegen gezeigt hätte. Dieses ethische Argument wird auch dafür bemüht, um vorzeitige Studienabbrüche (wegen nachgewiesener Überlegenheit einer der Alternativen) zu rechtfertigen. Ein solchermaßen «effizientes» Studiendesign ist aus verschiedenen Gründen gegebenenfalls auch finanziell effizient, woraus sich wieder die Implikationen mit der «Unabhängigkeit» ergeben.

Zur qualitativen Ergebnissicherheit (Verzerrungspotenzial) muss an dieser Stelle nicht (mehr) viel gesagt werden. Dafür ist der Umfang der Literatur zu diesem Thema zu groß, die Standards, die in der Vergangenheit gesetzt wurden, sind $\mathrm{zu}$ eindeutig, zu unumstritten, zu gut empirisch belegt [1, 24, 25]: Idealerweise ist eine Studie randomisiert (Vermeidung von Selektionsbias), doppelblind (Vermeidung von Durchführungs- und Entdeckungsbias), und alle Patienten gehen entsprechend ihrer randomisierten Therapiezuteilung in die Analyse ein (sogenanntes Intention-to-treat-Prinzip, Vermeidung von Abriebbias). Außerdem werden die Ergebnisse zu allen geplanten Endpunkten gemäß Studienprotokoll und statistischem Analyseplan publiziert (Vermeidung von Berichtsbias). Während die vollständige Berichterstattung immer und die Randomisierung bis auf extreme Ausnahmefälle ebenfalls immer realisiert werden können, müssen bei der Verblindung und bei der Umsetzung des Intention-to-treat-Prinzips zuweilen methodische Kompromisse eingegangen werden. So können nichtmedikamentöse Methoden häufig nicht den Anwendern gegenüber verblindet werden, aber eventuell bei den Patienten (und den die Patienten im weiteren Verlauf betreuenden Personen) oder zumindest denjenigen gegenüber, die die Zielkriterien einer Studie erheben. Und da es Realität in nahezu jeglichen Studien ist, dass nicht für alle Patienten alle Daten erhoben werden können (z.B. weil Patienten vorzeitig auf eigenen Wunsch aus einer Studie ausscheiden), müssen geeignete Ersetzungsstrategien für fehlende Werte a priori gefunden, geplant und dann auch angewendet werden. Die adäquate Auseinandersetzung mit diesen zentralen Designcharakteristika einer klinischen Studie und die konsequente Anwendung verzerrungsminimierender Strategien kennzeichnen eine gute klinische Studie im Hinblick auf die qualitative
Ergebnissicherheit. Studien erlauben auf dieser Basis vergleichsweise gesicherte Erkenntnisse oder eben nicht; ob sie auch noch «unabhängig» sind, ist dabei relativ uninteressant.

\section{Lücken und Herausforderungen}

Ein weiteres Moment einer guten klinischen Studie besteht aber in der Angemessenheit des Vergleichs. Es ist schon ethisch fragwürdig, z.B. ein neues Arzneimittel bei einer ernsthaften Erkrankung gegen ein Placebo zu testen, wenn bereits Therapien mit einem nachgewiesenen patientenrelevanten Nutzen verfügbar sind. Spätestens für Fragen einer angemessenen Versorgung werden darüber hinaus für neue medizinische Methoden Vergleiche mit dem jeweiligen Versorgungsstandard benötigt. Im Arzneimittelbereich stellt dies ein Problem dar, da die Zulassung auf europäischer Ebene multinational erteilt wird, in den einzelnen Mitgliedsstaaten der Europäischen Union (EU) aber unterschiedliche Versorgungsstandards existieren. Wenn also Arzneimittelstudien zum weit überwiegenden Teil von der pharmazeutischen Industrie finanziert werden, und zwar hauptsächlich zum Zwecke der Zulassung, dann fehlt es in der Tat an von der Industrie unabhängigen Studien, da deren Interesse an der Durchführung von Studien gegen Konkurrenzpräparate verständlicherweise begrenzt ist.

Und hier offenbart sich der Bereich, wo «Unabhängigkeit» doch interessant und relevant wird. Wichtige Fragen der medizinischen Anwendung, deren Beantwortung aber nicht im Interesse bestimmter Stakeholder liegt, werden nicht bearbeitet, jedenfalls solange nicht, wie nicht für diese Bearbeitung selbst wiederum (finanzielle) Anreize gesetzt werden. Eine Studie wie «Persephone» [26], in der die Halbierung der Therapiedauer mit Trastuzumab beim Brustkrebsrezidiv untersucht wird, liegt offensichtlich weit abseits der Interessen des Herstellers. Wenn dann auch kein regulatorisches Interesse (besser: regulatorischer Druck) besteht, ist die Klärung solcher Fragen nur möglich, wenn an der Untersuchung gleichzeitig ein großes Interesse der Anwender besteht und dazu passende Anreize, also Fördermöglichkeiten, geschaffen werden.

Interesse und Anreize bedingen sich hier aber gegenseitig. Auf Veranstaltungen werden oft die unzureichenden Fördermöglichkeiten beklagt und sie allein dafür verantwortlich gemacht, dass wichtige Fragen nicht bearbeitet und beantwortet werden können. Auch wenn zugestimmt wird, dass die Fördermöglichkeiten deutlich verbessert werden müssen, muss andererseits auch festgestellt werden, dass das Engagement vieler Anwender für das Durchführen guter, aussagefähiger Studien doch sehr verhalten ist. Aus vielen Stellungnahmen, die das IQWiG zu seinen Berichten erhält, ist neben sachlicher Detailkritik nicht selten eine generelle Ablehnung aussagekräftiger Studien (genauer natürlich: ihrer Ergebnisse) zu erkennen. Die Deutsche Gesellschaft für Nuklearmedizin (DGN) hat zum Beispiel gegen alle internationalen Standards 
ihre Position vertreten, dass «die Forderung nach randomisierten Outcome-Studien für das diagnostische Verfahren PET praxisfern ist und von der DGN abgelehnt wird» [27]. Eine solche Haltung, die deutlich macht, dass «Abhängigkeit» kein Industrieproblem ist, und die im Übrigen die nächsten 5 Jahre ohnehin nicht überleben wird, weil sie die Realität ignoriert [28], ist absolut kontraproduktiv, wenn es um Überzeugungsarbeit in Hinblick auf die bessere Förderung solcher Studien geht. Hierfür ist es erforderlich, laut und deutlich konkrete Forschungs- und Versorgungsprobleme zu benennen, die für ihre Bearbeitung erforderlichen und geeigneten guten Studien zu skizzieren, gegebenenfalls auch deutlich zu machen, wer und warum an einer Bearbeitung kein Interesse hat bzw. haben kann, sodass eine Beantwortung dieser Fragen im Interesse der Patienten nur durch eine «unabhängige» Finanzierung erfolgen kann und muss. Die Realisierung der PREFERE-Studie [20], die auf einen Bericht des IQWiG, viel Überzeugungsarbeit und umfangreiche Vorarbeiten des Instituts zusammen mit anderen Institutionen zurückgeht, zeigt, dass gute, wichtige, ja, sogar teure Studien in Deutschland finanziert und gemacht werden können.

Das IQWiG hält solche Studien für dringend notwendig und ist gerne bereit und dabei, zusammen mit Interessierten dafür zu werben, dass für wichtige Studienprojekte von klinischen Forschergruppen, die die Kriterien einer oben skizzierten «guten» patientenorientierten klinischen Studie erfüllen, angemessene Fördermöglichkeiten geschaffen werden.

\section{Disclosure Statement}

Die Autoren erklären, dass sie im Zusammenhang mit den Inhalten ihrer Arbeit keinerlei finanzielle Interessenkonflikte haben. Eine finanzielle Unterstützung durch Dritte erfolgte nicht. Die Autoren sind Mitarbeiter des Instituts für Qualität und Wirtschaftlichkeit im Gesundheitswesen und somit an der Generierung von klinischen Studien als der Grundlage der Institutsarbeit in höchstem Maße interessiert. Inwieweit sich daraus ein ideeller Interessenkonflikt ableiten lässt, muss der Einschätzung der Leserinnen und Leser der Arbeit vorbehalten bleiben.

\section{Literatur}

1 ICH Harmonised Tripartite Guideline. Statistical principles for clinical trials. International Conference on Harmonisation E9 Expert Working Group. Stat Med 1999;18:1905-1942.

2 Deutscher Bundestag: Gesetz zur Neuordnung des Arzneimittelmarktes in der gesetzlichen Krankenversicherung (Arzneimittelmarktneuordnungsgesetz - AMNOG). Bundesgesetzblatt 2010; Teil 1 (67):2262-2277.

3 Verordnung über die Nutzenbewertung von Arzneimitteln nach $\S 35$ a Absatz 1 SGB V für Erstattungsvereinbarungen nach $\S 130 \mathrm{~b}$ SGB V (Arzneimittel-Nutzenbewertungsverordnung - AM-NutzenV). Bundesgesetzblatt 2010; Teil 1(67):23242328.

4 Schott G, Gökbuget N, Pachl H, Ludwig WD: Klinische Studien in der Onkologie - Defizite und Lösungsvorschläge. Z Evid Fortbild Qual Gesundhwes 2011;105:657-664.

5 Wilmshurst P: The regulation of medical devices. Unsatisfactory, unscientific, and in need of a major overhaul. BMJ 2011;342:d2822.

6 Bose A, Hartmann M, Henkes H, Liu HM, Teng MM, Szikora I, Berlis A, Reul J, Yu SC, Forsting M, Lui M, Lim W, Sit SP: A novel, self-expanding, nitinol stent in medically refractory intracranial atherosclerotic stenoses: the Wingspan study. Stroke 2007:38:1531-1537.

7 Chimowitz MI, Lynn MJ, Derdeyn CP, Turan TN, Fiorella D, Lane BF, Janis LS, Lutsep HL, Barnwell SL, Waters MF, Hoh BL, Hourihane JM, Levy EI, Alexandrov AV, Harrigan MR, Chiu D, Klucznik RP, Clark JM, McDougall CG, Johnson MD, Pride GL Jr, Torbey MT, Zaidat OO, Rumboldt Z, Cloft HJ; SAMMPRIS Trial Investigators: Stenting versus aggressive medical therapy for intracranial arterial stenosis. N Engl J Med 2011;365:993-1003.

$>8$ Schünemann HJ, Oxman AD, Brozek J, Glasziou P, Jaeschke R, Vist GE, Williams JW Jr, Kunz R, Craig J, Montori VM, Bossuyt P, Guyatt GH; GRADE Working Group: Grading quality of evidence and strength of recommendations for diagnostic tests and strategies. BMJ 2008;336:1106-1110.
9 Gesetz zur Verbesserung der Versorgungsstrukturen in der gesetzlichen Krankenversicherung (GKV-Versorgungsstrukturgesetz - GKV-VStG). Bundesgesetzblatt 2011; Teil 1(70):2983-3022.

10 Gerdesmeyer L, Wagenpfeil S, Haake M, Maier M, Loew M, Wörtler K, Lampe R, Seil R, Handle G, Gassel S, Rompe JD: Extracorporeal shock wave therapy for the treatment of chronic calcifying tendonitis of the rotator cuff: a randomized controlled trial. JAMA 2003;290:2573-2580.

11 Schiener R, Brockow T, Franke A, Salzer B, Peter RU, Resch KL: Bath PUVA and saltwater baths followed by UV-B phototherapy as treatments for psoriasis: a randomized controlled trial. Arch Dermatol 2007;143:586-596.

12 Klein A, Schiffner R, Schiffner-Rohe J, EinseleKrämer B, Heinlin J, Stolz W, Landthaler M: A randomized clinical trial in psoriasis: synchronous balneophototherapy with bathing in Dead Sea salt solution plus narrowband UVB vs. narrowband UVB alone (TOMESA-study group). J Eur Acad Dermatol Venereol 2011;25:570-578.

13 Scharf HP, Mansmann U, Streitberger K, Witte S, Krämer J, Maier C, Trampisch HJ, Victor N: Acupuncture and knee osteoarthritis: a three-armed randomized trial. Ann Intern Med 2006;145:12-20.

14 Diener HC, Kronfeld K, Boewing G, Lungenhausen M, Maier C, Molsberger A, Tegenthoff M, Trampisch HJ, Zenz M, Meinert R; GERAC Migraine Study Group: Efficacy of acupuncture for the prophylaxis of migraine: a multicentre randomised controlled clinical trial. Lancet Neurol 2006;5:310-316.

15 Linde K, Streng A, Jürgens S, Hoppe A, Brinkhaus B, Witt C, Wagenpfeil S, Pfaffenrath V, Hammes MG, Weidenhammer W, Willich SN, Melchart D: Acupuncture for patients with migraine: a randomized controlled trial. JAMA 2005;293:21182125.

16 Witt C, Brinkhaus B, Jena S, Linde K, Streng A, Wagenpfeil S, Hummelsberger J, Walther HU, Melchart D, Willich SN: Acupuncture in patients with osteoarthritis of the knee: a randomised trial. Lancet 2005;366:136-143.
17 Treatment study of negative pressure wound therapy for diabetic foot wounds (DiaFu) [online]; in ClinicalTrials.gov 23.11.2011. http://clinicaltrials. gov/ct2/show/NCT01480362 (letzter Zugriff am 27. August 2012).

18 Treatment study of vacuum assisted closure for postsurgical subcutaneous abdominal wound healing impairments (SAWHI) [online]; in ClinicalTrials.gov 02.02.2012. http://clinicaltrials.gov/ct2/show/ NCT01528033 (letzter Zugriff am 27. August 2012).

19 Haematopoietic stem cell transplantation (HSCT) in comparison to conventional consolidation therapy for patients with acute myeloid leukemia (AML) (intermediate risk) $<=60 \mathrm{y}$. after first CR [online]; in ClinicalTrials.gov 22.11.2010. http:// clinicaltrials.gov/ct2/show/NCT01246752 (letzter Zugriff am 27. August 2012).

20 Stöckle M, Bussar-Maatz R: Organbegrenztes Prostatakarzinom - PREFERE-Studie. Z Evid Fortbild Qual Gesundhwes 2012;106:333-335.

21 Jekerle V, Schröder C, Pedone E: Legal basis of the Advanced Therapies Regulation. Bundesgesundheitsblatt Gesundheitsforschung Gesundheitsschutz 2010;53:4-8.

22 European Medicines Agency: CAT monthly report of application procedures, guidelines and related documents on advanced therapies. July 2012 Meeting. EMA/CAT/497804/2012.

23 Bührlen B, Vollmar HC: Büro für Technologiefolgen-Abschätzung beim Deutschen Bundestag. Biomedizinische Innovationen und klinische Forschung - Wettbewerbs- und Regulierungsfragen. Innovationsreport. Arbeitsbericht 2009; Nr 132.

24 Higgins JP, Altman DG, Gøtzsche PC, Jüni P, Moher D, Oxman AD, Savovic J, Schulz KF, Weeks L, Sterne JA; Cochrane Bias Methods Group; Cochrane Statistical Methods Group: The Cochrane Collaboration's tool for assessing risk of bias in randomised trials. BMJ 2011;343:d5928. 
25 Guyatt GH, Oxman AD, Vist G, Kunz R, Brozek J, Alonso-Coello P, Montori V, Akl EA, Djulbegovic B, Falck-Ytter Y, Norris SL, Williams JW Jr, Atkins D, Meerpohl J, Schünemann HJ: GRADE guidelines: 4 . Rating the quality of evidence - study limitations (risk of bias). J Clin Epidemiol 2011;64: 407-415.
26 Persephone: Duration of herceptin with chemotherapy 6 versus 12 months [online]; in Controlledtrials.com 09.02.2007. www.controlled-trials.com/ ISRCTN52968807/ (letzter Zugriff am 27. August 2012)

27 Kotzerke J, Dietlein M, Grünwald F, Bockisch A: Vorbehalte der DGN zur Nutzenbewertung der PET durch das IQWiG. Nuklearmedizin 2010;49:612.
8 Scheibler F, Zumbé P, Janssen I, Viebahn M, Schröer-Günther M, Grosselfinger R, Hausner E, Sauerland S, Lange S: Randomized controlled trials on PET: A systematic review of topics, design, and quality. J Nucl Med 2012;53:1016-1025. 